\title{
Estudo fitoquímico e avaliação das atividades moluscicida e larvicida dos extratos da casca do caule e folha de Eugenia malaccensis L. (Myrtaceae)
}

\author{
Anderson Marques de Oliveira ${ }^{1}$, Margarida Maria dos Santos Humberto ${ }^{1}$, José Marcos da \\ Silva', Rosário de Fátima de Almeida Rocha², Antônio Euzébio Goulart Sant'Ana ${ }^{1 *}$ \\ ${ }^{1}$ Instituto de Química e Biotecnologia, Universidade Federal de Alagoas, Cidade Universitária, \\ 57072-970, Maceió, AL, Brasil, \\ ${ }_{2}^{2}$ Instituto de Ciências Biológicas e Básicas da Saúde, Universidade Federal de Alagoas, Cidade Universitária, \\ 57000-000, Maceió, AL, Brasil
}

\begin{abstract}
RESUMO: Eugenia malaccensis L. (Syzygium malaccensis Merr.\& Perry), denominada comumente de jambo vermelho, é uma espécie arbórea de ocorrência comum no Brasil e faz parte das plantas medicinais do Estado de Alagoas. Estudos químicos anteriores com E. malaccensis foram realizados com plantas coletadas na Ásia, Oceania e na América Central. Este trabalho descreve o estudo químico do extrato etanólico das folhas e cascas de um espécimem coletado no Estado de Alagoas, Brasil. Considerando-se a escassa literatura sobre o potencial moluscicida e larvicida dessa espécie, buscou-se testar estas atividades contra caramujos Biomphalaria glabrata Say, e frente às larvas do mosquito Aedes aegypti L. Os extratos etanólicos da casca do caule e das folhas foram inativos frente às larvas de Aedes aegypti na concentração de $500 \mu \mathrm{g} / \mathrm{mL}$, porém, ativos contra Biomphalaria glabrata $\left(\mathrm{CL}_{50}=42,53\right.$ e $41,90 \mu \mathrm{g} / \mathrm{mL}$, respectivamente). O estudo químico levou ao isolamento e identificação de 5,7,3',4',5'-penta-hidroxi-flavonol (1), 5,7,3',5'tetra-hidroxi-4'-metoxi-flavonol (2), ácido 3,4,5-tri-hidroxibenzóico (3) e ácido 3-acetil-urs-12en-28-óico (4) das folhas e da casca do caule. O composto 4 é isolado pela primeira vez no gênero Eugenia. As estruturas desses compostos foram estabelecidas através de estudos no IV, de RMN ${ }^{1} \mathrm{H}$ $\mathrm{e}^{13} \mathrm{C}$ e comparação com dados da literatura.
\end{abstract}

Unitermos: Biomphalaria glabrata, Eugenia malaccensis, Myrtaceae, flavonóis, ácido 3-acetilurs-12-en-28-óico.

\begin{abstract}
Phytochemical studies of the extracts of stem bark and leaves of Eugenia malaccensis L. (Myrtaceae) and evaluation of their molluscicidal and larvicidal activities". Eugenia malaccensis L. (Syzygium malaccensis Merr. \& Perry), popularly known in Brazil as jambo vermelho, is a very common tree and one of the medicinal plants of Alagoas State, Brazil. Previous chemical studies with $E$. malaccensis have been carried out with native plants from Asia, Oceania, and Central America. This work describes the chemical study of E. malaccensis L., a specimen collected in Alagoas State. Considering that studies on the molluscicidal and larvicidal potential of E. malaccensis were not reported, this work aimed to test its molluscicidal activity against Biomphalaria glabrata snails and larvicidal activity against Aedes aegypti mosquitos. The ethanolic stem bark and leaf extracts were inactive towards Aedes aegypti larvae. The ethanolic stem bark and leaf extract were active against Biomphalaria glabrata with $\mathrm{CL}_{50}=42.53$ and 41.90 $\mu \mathrm{g} / \mathrm{mL}$, respectively. 5,7,3',4',5'-Penta-hydroxy-flavonol (1), 5,7,3',5'-tetra-hydroxy-4'-methoxyflavonol (2), 3,4,5-tri-hydroxybenzoic acid (3) and 3-acetyl-urs-12-en-28-oic acid (4) were isolated

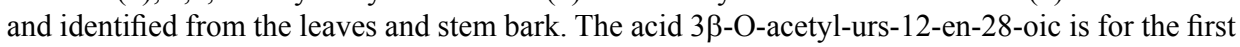
time isolated from the genus Eugenia. The structures of the compounds have been established by IR, ${ }^{1} \mathrm{H}$ and ${ }^{13} \mathrm{C}$ NMR spectral analysis and by comparison with literature data.
\end{abstract}

Keywords: Biomphalaria glabrata, Eugenia malaccensis, Myrtaceae, flavonols, 3-acetyl-urs-12en-28-oic acid.

\section{INTRODUÇÃO}

A família Myrtaceae compreende cerca de 80 gêneros, com aproximadamente 3.000 espécies de árvores e arbustos, largamente espalhadas nas florestas brasileiras e distribuídas em regiões tropicais e subtropicais do globo
(Vieira et al., 2004; De Oliveira et al., 2005). Muitas de suas espécies são cultivadas, seja por conta dos frutos comestíveis, seja com finalidade ornamental ou, ainda, visando-se a extração de essências de valor comercial (Joly, 1993; Vieira et al., 2004; Silva et al., 2005a). Esta família está dividida em duas subfamílias: Myrtoideae e 
Leptospermoideae e, no Brasil, todos os representantes nativos pertencem à subfamília Myrtoideae, onde se inclui o gênero Eugenia (Limberger et al., 2004).

O gênero Eugenia é considerado um dos maiores da família Myrtaceae, com aproximadamente 500 espécies de árvores e arbustos, dos quais cerca de 400 distribuemse no Brasil (De Oliveira et al., 2005) e assumem destaque especial, por fornecer extratos utilizados na medicina tradicional como agentes antimicrobianos, antiinflamatórios (Hussein et al., 2003), antidiabéticos, nas arritmias ou insuficiência cardíaca (Vendruscolo et al., 2005) entre outras aplicações (Timbola et al., 2002). No Brasil, entre as espécies populares e apreciadas encontram-se a jaboticabeira (E. edulis Vell.) (Hussein et al., 2003), a pitangueira (E. uniflora L.) (Auricchio; Bacchi, 2003), o jambolão (E. jambolana Lam.) (Alberton et al., 2001; Pepato et al., 2005) e o cravo da Índia (E. caryophyllata Thunb.) (Corrêa, 1984; Kelecom et al., 2002). Estudos químicos com espécies de Eugenia revelaram, sobretudo, a presença de flavonóides, taninos, terpenóides e óleos essenciais (Lunardi et al., 2001; Mahmoud et al., 2001; Frighetto et al., 2005; Oliveira et al., 2005), enquanto, sob o ponto de vista farmacológico, estudos realizados com extratos brutos e compostos, comprovaram as atividades antiinflamatória, analgésica, antifúngica, antipirética (Kuskoski et al., 2003; Falcão et al., 2005; Lima et al., 2006), hipotensiva (Consolini et al., 1999), antidiabética (Ravi et al., 2005; Barbosa-Filho et al., 2005) e antioxidante (Velazquez et al., 2003).

A espécie E. malaccensis L. (sinonímia: Syzygium malaccense Merr. \& Perry), conhecida popularmente no Brasil como jambo vermelho, jambo roxo e jambo encarnado, faz parte das plantas medicinais do Estado de Alagoas, sendo as folhas e os frutos usados como adstringentes e estimulantes do apetite, como diuréticos e no combate a anemias, principalmente pelas populações de baixo poder aquisitivo (Campelo, 1988). Em várias partes do mundo, E. malaccense, é empregada, na medicina popular, no tratamento de processos inflamatórios, febre, coceira, diabetes, catarro no pulmão, tosse, dores de cabeça, disenteria e como diurético (Dunstan et al. 1997; Noreen et al. 1998). Estudos farmacológicos demonstraram a atividade antiviral seletiva do extrato aquoso da casca do caule frente aos vírus da Herpes Simplex-1 (HSV-1) e 2 (HSV-2), da estomatite vesicular e HIV-1 (Locher et al., 1995; 1996), enquanto, o extrato aquoso das folhas revelou forte inibição das bactérias Staphylococcus aureus e do Streptococcus pyogenes (Locher et al., 1995) tendo sido isolado o ácido elágico, o 3-O-metilelágico e o 3,3'-O-dimetilelágico.

Estudos químicos efetuados com as folhas de $E$. malaccensis levaram ao isolamento de quatro flavonóides: catequina, mearnsitrina, miricitrina e quercitrina (Noreen et al., 1998); dos frutos foram obtidos compostos voláteis (Pino et al., 2004). A partir das flores foi isolada a antocianina, 3,5-diglicosílmalvidina (Lowry, 1976a), enquanto do caule foram isolados os ácidos elágico, 3-O- metilelágico e 3,3'-O-dimetilelágico (Lowry, 1968). Cabe ressaltar, porém, que os referidos estudos fitoquímicos encontrados na literatura são abordados com plantas cultivadas em Cuba (Pino et al., 2004), Singapura (Lowry et al., 1976), Samoa (Noreen et al., 1998) e Malásia (Lowry, 1968). Tendo em mente a possível variabilidade da composição química devido a fatores ambientais $\mathrm{e}$ sazonais, optou-se por investigar a composição química dos extratos das folhas e das cascas do caule do jambo cultivado no Brasil e avaliar o seu potencial moluscicida e larvicida .

A esquistossomose e o dengue são dois importantes problemas de saúde pública que muitos países da América Latina Tropical, incluindo grande parte do Nordeste do Brasil, buscam solucionar. O uso de derivados de plantas para controle ou erradicação dessas doenças, aliado à imediata disponibilidade dessas plantas nas áreas afetadas são de grande valor, já que a maior parte da população que vive nessas áreas encontra nos produtos naturais, especialmente nas plantas, a única fonte de recursos terapêuticos disponíveis (Simas et al., 2004; Luna et al., 2005; Ruiz et al., 2005).

\section{MATERIAL E MÉTODOS}

\section{Métodos gerais}

As cromatografias em coluna foram efetuadas em gel de sílica (70-230 e 230-400 mesh, Vetec, Rio de Janeiro, RJ. Brasil) e em Sephadex LH-20 (Amersham Pharmacia Biotech AB, Uppsala, Suécia). Os espectros no IV foram registrados em pastilhas de $\mathrm{KBr}$ utilizando espectrofotômetro Perkin-Elmer, FT-IR-1600.(Perkin Elmer, Palo Alto CA. USA) Os espectros de RMN $\left({ }^{1} \mathrm{H}: 250\right.$ e $400 \mathrm{MHz} ;{ }^{13} \mathrm{C}$ : 62,9 e $100 \mathrm{MHz}$ ) foram obtidos em espectrômetros BRUKER (Bruker Analytic, Ettlingen, Alemanha) modelos AC-250 e ARX-400, respectivamente. O sinal residual do solvente ou TMS foi utilizado como referência interna.

\section{Coleta e identificação do material vegetal}

As folhas e cascas do caule de um espécime Eugenia malaccensis L. foram coletadas e identificadas pela botânica Prof ${ }^{a}$ Rosário de Fátima de Almeida Rocha, do Departamento de Botânica do Instituto de Ciências Biológicas e Básicas da Saúde da Universidade Federal de Alagoas, em setembro de 2003, no campus A.C. Simões da Universidade Federal de Alagoas, localizado no município de Maceió, Estado de Alagoas (Brasil). Um exemplar do referido material encontra-se catalogado ( $\mathrm{N}^{\circ}$ 003803), no Herbário Professor Honório Monteiro, do Departamento de Botânica do Instituto de Ciências Biológicas e Básicas da Saúde da UFAL, sediado no Museu de História Natural (MUFAL).

\section{Extração e isolamento dos constituintes químicos}


Tabela 1. Atividades larvicida (Aedes aegypti) e moluscicida (caramujos adultos - Biomphalaria glabrata) dos extratos etanólicos das cascas do caule e das folhas de Eugenia malaccensis

\begin{tabular}{llcc}
\hline Parte & Extrato & A. aegypti $^{a}(\mu \mathrm{g} / \mathrm{mL})$ & B.glabrata $^{b} \mathrm{CL}_{50}$ \\
\hline Cascas do Caule & Etanólico & inativo & 42,53 \\
Folhas & Etanólico & inativo & 41,90 \\
& & & \\
\hline
\end{tabular}

${ }^{a}$ concentração de $500 \mu \mathrm{g} / \mathrm{mL},{ }^{b}(\mu \mathrm{g} / \mathrm{mL})$<smiles>[R2]Oc1c(O)cc(-c2oc3cc(O)cc(O)c3c(=O)c2O)cc1O</smiles>

Figura 1. Substâncias isoladas de Eugenia malaccensis

As folhas e cascas do caule de E. malaccensis foram secas espontaneamente à sombra e submetidas à moagem. O pó das cascas do caule $(700 \mathrm{~g})$ e das folhas $(600 \mathrm{~g})$ foi submetido a extração com etanol $(4 \mathrm{x} 2 \mathrm{~L}$, 72 h de maceração cada). Após remoção do solvente em evaporador rotatório, foram obtidos $72,2 \mathrm{~g}$ de extrato etanólico bruto de caule e $86,6 \mathrm{~g}$ de extrato etanólico bruto de folhas. Parte dos dois extratos $(65,2 \mathrm{~g}-$ caule, 70,5 - folhas) foi, isoladamente, solubilizada com $\mathrm{MeOH}-$ $\mathrm{H}_{2} \mathrm{O}(3: 2)$ e extraída sucessivamente com $n-\mathrm{C}_{6} \mathrm{H}_{14}, \mathrm{CHCl}_{3}$ e AcOEt (4 x $250 \mathrm{~mL}$ cada). A fração em AcOEt das folhas $(9,5 \mathrm{~g})$ foi submetida a sucessivos fracionamentos cromatográficos em coluna de gel de sílica e misturas de $\mathrm{CHCl}_{3}$, AcOEt e MeOH como eluentes. As frações obtidas foram comparadas por cromatografia em camada delgada de sílica usando-se como eluente a mistura hexano : acetato de etila : etanol $(50: 45: 5)$ e como revelador solução ácida de sulfato cérico, reunindo-se as semelhantes. A fração eluída da coluna com AcOEt foi submetida à cromatografia em coluna, utilizando-se Sephadex LH-20 como suporte e metanol como eluente. O procedimento resultou no isolamento de dois flavonóis, miricetina (25 mg) (1) e mearnsetina (110 mg) (2), e do ácido gálico $(120 \mathrm{mg})(3)$. A fração em $\mathrm{C}_{6} \mathrm{H}_{14}$ das cascas do caule $(5,0 \mathrm{~g})$, após sucessivos fracionamentos cromatográficos em coluna de gel de sílica, resultou no isolamento do ácido 3-acetil ursólico (20 mg) (4).

Miricetina (5,7,3'4',5'-penta-hidroxi-flavonol) (1). Aspecto físico: sólido amorfo amarelo. IV $(\mathrm{KBr}) \mathrm{cm}^{-}$ ${ }^{1}: 3408,1660,1598,1520,1464,1329,1027 . \mathrm{RMN}{ }^{1} \mathrm{H}$ $\left(400 \mathrm{MHz}, \mathrm{CDCl}_{3}\right): \delta 6,37(1 \mathrm{H}, \mathrm{d}, J=2,0 \mathrm{~Hz}, \mathrm{H}-6) ; 6,56$ $(1 \mathrm{H}, \mathrm{d}, J=2,0 \mathrm{~Hz}, \mathrm{H}-8) ; 7,53$ (2H, s, H-2', 6'). ${ }^{13} \mathrm{C}-\mathrm{APT}$
(100 MHz, $\left.\mathrm{CDCl}_{3}\right): \delta 94,64(\mathrm{C}-8) ; 99,48(\mathrm{C}-6) ; 104,77$ (C-10); 108,78 (C-2', 6'); 121,36 (C-1'); 137,21 (C-3); 137,65 (C-4'); 147,00 (C-3', 5'); 148, 27 (C-2); 158,47 (C-9); 162,78 (C-5); 165, 85 (C-7); 177,57 (C-4).

Mearnsetina (5,7,3',5'-tetra-hidroxi-4'-metoxiflavonol) (2). Aspecto físico: sólido amorfo amarelo. IV (KBr) $\mathrm{cm}^{-1}: 3388,1658,1617,1506,1452,1380,1203$, 1025. RMN ${ }^{1} \mathrm{H}\left(400 \mathrm{MHz}, \mathrm{CDCl}_{3}\right): \delta 4,05\left(3 \mathrm{H}, \mathrm{s}, \mathrm{OCH}_{3}\right)$; $6,37(1 \mathrm{H}, \mathrm{d}, J=2,0 \mathrm{~Hz}, \mathrm{H}-6) ; 6,56(1 \mathrm{H}, \mathrm{d}, J=2,0 \mathrm{~Hz}, \mathrm{H}-$ 8); 7,49 (2H, s, H-2',6'). ${ }^{13} \mathrm{C}-\mathrm{APT}\left(100 \mathrm{MHz}, \mathrm{CDCl}_{3}\right): \delta$ 61,11 $\left(\mathrm{OCH}_{3}\right)$; 94,68 (C-8); 99,58 (C-6); 104,83 (C-10); 108,90 (C-2', 6'); 128,27 (C-1'); 138,37 (C-3); 138,73 (C-4'); 147,27 ( C-2); 151,95 (C-3', 5'); 158,54 (C-9); 162,82 (C-5 ); 166,07 (C-7); 177,78 (C-4).

Ácido gálico (Ácido 3,4,5 tri-hidroxibenzóico (3). Aspecto físico: cristal branco. P.F. $254-255^{\circ} \mathrm{C}(\mathrm{MeOH})$; (lit: $251-252^{\circ} \mathrm{C}$; De Almeida et al., 2005). IV (KBr) $\mathrm{cm}^{-1}$ : 3477, 3200-2500, 1700, 1623, 1548, 1455, 1273, 1044, 867. RMN ${ }^{1} \mathrm{H}\left(250 \mathrm{MHz}, \mathrm{CD}_{3} \mathrm{OD}\right): \delta$ 7,09 (s, 2H). RMN ${ }^{13} \mathrm{C}$ (62,9 MHz, CD $\left.\mathrm{OD}\right): \delta 110,42$ (C-2, C-6); 122,05 (C1); 139, 63 (C-4 ); 146,41 (C-3, C-5); 170,50 (C-7).

Ácido 3ß-O-acetil-urs-12-en-28-óico (4). Aspecto físico: sólido amorfo branco. P.F. $210-214^{\circ} \mathrm{C}$ (MeOH); (lit: $214-217^{\circ} \mathrm{C}$; Vieira et al., 2004). IV (KBr) $\mathrm{cm}^{-1}:$ 3200-2600, 1734, 1693, 1449, 1372, 1243, 1026. $\mathrm{RMN}{ }^{1} \mathrm{H}\left(400 \mathrm{MHz}, \mathrm{CDCl}_{3):} \delta\right.$ 0,80 (s, H-25); 0,88 (s, H-24); 0,89 (s, H-23); 0,90 (d, $J=6,7 \mathrm{~Hz}, \mathrm{H}-29) ; 0,98$ (d, $J=6,4$ Hz, H-30); 0,99 (s, H-26); 1,11 (s, H-27); 2,06 (s, H-32); 4,53 (tl, $J=7,6 \mathrm{~Hz}, \mathrm{H}-3) ; 5,28$ (t, $J=3,5 \mathrm{~Hz}, \mathrm{H}-$ 12). $\mathrm{RMN}{ }^{13} \mathrm{C}\left(100 \mathrm{MHz}, \mathrm{CDCl}_{3}\right): \delta 15,51(\mathrm{C}-25) ; 16,69$ (C-24); 17,00 (C-29); 17,08 (C-26); 18,14 (C-6); 21,16 (C-32); 21,30 (C-30); 23,26 (C-11); 23,37 (C-2); 23,55 
(C-27); 24,05 (C-16); 27,95 (C-15); 28,05 (C-23); 30,57

(C-21); 32,81 (C-7); 36,69 (C-22); 36,88 (C-10); 37,85

(C-4); 38,24 (C-1); 38,80 (C-20); 38,99 (C-19); 39,46

(C-8); 41,89 (C-14); 47,43 (C-9); 47,92 (C- 17); 52,53

(C-18); 55,27 (C-5); 80,91 (C-3); 125,72 (C-12); 137,90

(C-13); 171,30 (C-31); 182,90 (C-28).

\section{Avaliação da atividade moluscicida}

Os moluscos utilizados da espécie Biomphalaria glabrata, com diâmetro entre $13-18 \mathrm{~mm}$, provenientes do Instituto René Rachou, de Belo Horizonte (MG), foram mantidos em aquários sob circulação contínua de água desclorada e a uma temperatura de $28{ }^{\circ} \mathrm{C}$, no laboratório de bioensaios da UFAL. A metodologia utilizada está de acordo com a descrita na literatura (Dos Santos; Sant'Ana 1999). Foram testados os extratos etanólicos das cascas do caule e das folhas. $\mathrm{O}$ ensaio consistiu na imersão do caramujo em uma solução aquosa a $0,1 \%$ de DMSO do extrato ou fração sob investigação, por 24 horas, nas concentrações apropriadas. Decorrido esse período, os moluscos foram lavados, introduzidos em água desclorada e alimentados, permanecendo sob observação por mais 24 horas, com registro e retirada dos caramujos mortos.

Nos bioensaios foram utilizados cinco caramujos em $125 \mathrm{~mL}$ de solução, em concentrações que variaram de $100 \mu \mathrm{g} / \mathrm{mL}$ a $10 \mu \mathrm{g} / \mathrm{mL}$, sendo cada concentração testada em duplicata. Os extratos que apresentaram atividade moluscicida em concentrações abaixo de 100 $\mu \mathrm{g} / \mathrm{mL}$, nos bioensaios, foram considerados ativos e submetidos a testes posteriores onde foram utilizados 10 caramujos, mantendo sempre a relação de $25 \mathrm{~mL}$ de solução por caramujo, sendo cada concentração testada em triplicata. Paralelamente, foram realizados testes de controle com solução aquosa de dimetilsulfóxido a 0,1 \% e com o moluscicida Niclosamida ${ }^{\circledR}$ a $3 \mu \mathrm{g} / \mathrm{mL}$. Para cálculo de $\mathbf{C L}_{50}$ foi utilizado o programa Probit versão 1.5 (EPA, 2004).

\section{Avaliação da atividade larvicida}

Os testes com as larvas dos mosquitos Aedes aegypti foram realizados no laboratório de bioensaios do Instituto de Química e Biotecnologia da UFAL, de acordo com a metodologia preconizada pela OMS (WHO, 1975). Foram preparadas soluções com água destilada a $1 \%$ de DMSO dos extratos etanólicos das cascas do caule e das folhas de E. malaccensis, na concentração inicial de 500 $\mu \mathrm{g} / \mathrm{mL}$. Foram utilizadas larvas de $A$. aegypti do quarto estádio em quadruplicata. Cada parcela experimental constou de 10 larvas, que foram postas em contato com 20 $\mathrm{mL}$ de solução teste. A contagem das larvas foi realizada a $24 \mathrm{~h}$ e $48 \mathrm{~h}$ do início do experimento. Paralelamente, foram realizados testes de controle com solução aquosa de DMSO a $1 \%$ e com o larvicida sintético, Temephos ${ }^{\circledR}$ a $3 \mu \mathrm{g} / \mathrm{mL}$.

\section{RESULTADOS E DISCUSSÃO}

A substância 1 apresentou, em seu espectro no IV, absorções em 3408 e $1329 \mathrm{~cm}^{-1}$, sugerindo a presença de grupo $\mathrm{OH}$ de fenol e/ou de álcool. A presença de carbonila conjugada foi evidenciada pela absorção forte em $1660 \mathrm{~cm}^{-1}$, enquanto a natureza aromática foi revelada pelas bandas em 1598, 1520 e $1464 \mathrm{~cm}^{-1}$, correspondentes às deformações axiais das ligações $\mathrm{C}=\mathrm{C}$ do anel aromático (Silverstein; Basser, 1994). O espectro de $\mathrm{RMN}{ }^{1} \mathrm{H}$ da substância $\mathbf{1}$ exibiu sinais apenas na região de hidrogênios aromáticos, dentre eles um simpleto em $\delta$ 7,53 , integrando para dois hidrogênios. Outros dois sinais na forma de dupletos em $\delta 6,37$ e $\delta 6,56$, integrando para um hidrogênio cada e $J=2,0 \mathrm{~Hz}$, são característicos de acoplamento em anéis meta substituídos. Comparando-se estes valores, aliados aos dados fornecidos pelo espectro no IV, com espectros de flavonóis já descritos (Silva et al., 2005b; Zhang et al., 2005), pôde-se propor um esqueleto desta classe para a substância 1. O par de dupletos mostrou tratar-se das absorções dos hidrogênios 6 e 8 do anel A do esqueleto de flavonóides 5,7-di-hidroxilados, enquanto o simpleto em $\delta$ 7,53 foi atribuído aos hidrogênios 2' e 6', de acordo com um padrão de trissubstituição (3', 4',5') no anel B (Sharaf et al., 1999).

A análise dos espectros de RMN ${ }^{13} \mathrm{C}$-APT e HMQC de 1 revelou um total de 13 sinais, dos quais, nove referem-se a carbonos não hidrogenados e os outros quatro, a cinco carbonos monoidrogenados. Os sinais em $\delta 177,57$, $\delta 148,27$ e $\delta 137,21$ correspondem, respectivamente, aos carbonos C-4, C-2 e C-3 do esqueleto do flavonol (Hussein et al., 2003), como sugerido no espectro de RMN ${ }^{1} \mathrm{H}$. A análise dos dados espectrais, bem como as comparações com a literatura permitiram identificar a substância $\mathbf{1}$ como o 5,7,3',4',5'-penta-hidroxiflavonol, conhecido como miricetina, já descrito na literatura (Hussein et al., 2003), e, ainda, isolado como 3-O- $\alpha$-L-ramnosídeo das folhas de um espécime de E. malaccensis (Noreen et al., 1998). Essa substância possui reconhecida ação antiinflamatória, antiosteoporótica, antiarteriosclerótica, antiviral, antioxidante, anticarcinogênica, antidiabética, antitrombose, antimicrobiana e moluscicida (Ong; Khoo, 1997; Carvalho et al., 2000; Kuo, 2005; Barbosa et al., 2006).

O espectro de RMN ${ }^{1} \mathrm{H}$ da substância 2 difere basicamente daquele do flavonol 1, devido à presença de um sinal adicional, na forma de um simpleto em $\delta$ 4,05 , integrado para três hidrogênios, atribuível a um grupo metoxila. Já o espectro de RMN ${ }^{13} \mathrm{C}$-APT de 2 revelou um total de 14 sinais, dos quais o sinal adicional, em $\delta 61,11$, relativamente ao espectro de $\mathbf{1}$, confirma a ocorrência de grupo metoxila. Comparando-se os valores de deslocamentos químicos entre as substâncias $\mathbf{1}$ e $\mathbf{2}$ pôde-se concluir que ambas detêm o mesmo padrão de substituição nos anéis A e C. Um valor de deslocamento químico acima de $\delta 60,00$ para grupo metoxila é um indicativo de que esse grupo encontra-se em uma relação

Rev. Bras. Farmacogn. Braz J. Pharmacogn. 16(Supl.):dez. 2006 
orto a dois grupos oxigenados (Noreen et al., 1998; Hussein et al., 2003). Isto permitiu inferir a metoxila na posição C-4'. A análise dos dados espectrais, bem como as comparações com a literatura permitiram identificar a substância 2 como sendo o 5,7,3',5'-tetra-hidroxi-4'metoxiflavonol, conhecido como mearnsetina (2), já descrito na literatura (Noreen et al., 1998) e, também, isolado como 3-O- $\alpha$-L-ramnosídeo das folhas de $E$. malaccensis (Noreen et al., 1998).

A substância 3 apresentou, em seu espectro no IV, bandas em 3477 e $1273 \mathrm{~cm}^{-1}$, sugerindo grupo $\mathrm{OH}$ de fenol. Absorções para deformação axial $\mathrm{C}=\mathrm{C}$ de aromáticos foram observadas em 1623, 1548, $1455 \mathrm{~cm}^{-}$ 1, além de absorções de grupo carboxila, sugerida pela larga banda de deformação axial de O-H em 3200-2500 $\mathrm{cm}^{-1}$, juntamente com uma forte absorção em $1700 \mathrm{~cm}^{-}$ 1 , relativa à deformação axial de $\mathrm{C}=\mathrm{O}$ de carbonila. $\mathrm{O}$ espectro de $\mathrm{RMN}{ }^{1} \mathrm{H}$ de 3 exibiu apenas um sinal, na forma de simpleto em $\delta 7,09$, condizente com hidrogênio aromático. Já, o espectro de $\mathrm{RMN}{ }^{13} \mathrm{C}$ revelou 5 sinais, correspondentes a 7 átomos de carbono. O sinal em $\delta 170,50$ foi atribuído a grupo carboxila, conforme evidenciado no espectro na região do IV. Os quatro sinais restantes apresentam valores de deslocamentos químicos compatíveis com carbonos aromático $(\delta 110,42$; $122,05 ; 139,63 ; 146,41)$. Com base nessas informações, associadas à análise comparativa com a literatura (De Almeida et al., 2005), foi atribuída a estrutura do ácido 3,4,5-tri-hidroxibenzóico, conhecido como ácido gálico, para a substância 3 , sendo aqui descrito pela primeira vez nesta espécie.

Para o ácido gálico foram descritas as ações anticarcinogênica, antimutagênica, antioxidante, antialérgica, antiinflamatória, antifúngica e inibidor da enzima conversora de angiotensina (Negi et al., 2005; Barbosa-Filho et al., 2006b). Além dessas atividades, o ácido gálico é usado como protótipo, originando diversos derivados com importantes ações farmacológicas, tais como antitumorais, inibidora do HIV-1, antioxidantes e antimalariais (Pellegrina et al., 2005).

A natureza triterpênica da substância 4 (Figura 1) foi sugerida por cromatografia em camada delgada de sílica, pela coloração rósea-avermelhada frente ao reagente de Liebermann-Burchard e coloração violeta com solução de sulfato cérico (Jain; Yadava, 1994). $\mathrm{Na}$ análise do espectro na região do IV de $\mathbf{4}$ foram observadas, dentre outras, duas bandas em 1734 e $1693 \mathrm{~cm}^{-1}$, compatíveis com grupos carbonila de éster e ácido carboxílico, respectivamente. A primeira foi corroborada pelas absorções intensas em 1243 e 1026 $\mathrm{cm}^{-1}$, correspondentes às deformações axiais de $\mathrm{CO}$, e a segunda pôde ser inferida pela banda larga entre 3200$2600 \mathrm{~cm}^{-1}$, correspondente à deformação axial de $\mathrm{OH}$ (Silverstein; Brassler, 1994). O espectro de RMN ${ }^{1} \mathrm{H}$ de 4 exibiu um sinal na forma de tripleto em $\delta \quad 5,28$ $(J=3,5 \mathrm{~Hz}, 1 \mathrm{H})$, indicativo de hidrogênio olefínico $\mathrm{H}-$ 12 de triterpeno pentacíclico com esqueleto urs-12-eno

(David et al., 2004). Esterificação em C-3 do esqueleto triterpênico foi sugerida pela presença de um tripleto largo em $\delta 4,53$, integrando para um hidrogênio, com constante de acoplamento de $J=7,6 \mathrm{~Hz}$. Essa observação foi confirmada pela ocorrência de um sinal simples em $\delta 2,06$, integrado para três hidrogênios, característico de grupo acetoxila em C-3 (David al., 2004). Dentre outros sinais estão presentes aqueles correspondentes a 7 grupos metila do núcleo triterpênico $(\delta 1,11$ a 0,80$)$. A análise dos espectros de $\mathrm{RMN}{ }^{13} \mathrm{C}$-APT revelou 32 sinais; entre eles destacam-se os sinais referentes aos carbonos do grupo carboxila em $\delta 182,90$ e do grupo acetoxila em $\delta$ 171,30, confirmando as informações dos espectros IV e RMN ${ }^{1} \mathrm{H}$. Os dois sinais em $\delta 125,72(\mathrm{CH})$ e $137,90(\mathrm{C})$ são característicos de carbonos olefínicos C-12 e C-13 de esqueleto urs-12-eno (Santos et al., 1997; David et al., 2004). A comparação com os valores de $\mathrm{RMN}{ }^{13} \mathrm{C}$ da literatura, empregando dois modelos de triterpenos como referência, a $\alpha$-amirina e o ácido $3 \beta$ - $O$-trans- $p$-cumaroilurs-12-en-28-óico (David et al., 2004) permitiu atribuir os valores dos deslocamentos químicos dos carbonos e determinar a estrutura da substância $\mathbf{4}$ como sendo o ácido $3 \beta$ - $O$-acetilurs-12-en-28-óico, conhecido como acetato do ácido ursólico (Vieira et al., 2004; Takeda et al., 2004). $\mathrm{Na}$ família Myrtaceae, foi encontrado apenas um relato de sua ocorrência na espécie Malaleuca alternifolia (Vieira et al., 2004), sendo, portanto, o primeiro relato no gênero Eugenia (Frighetto et al., 2005).

O ácido ursólico e alguns derivados foram isolados anteriormente de várias espécies de Eugenia (Frighetto et al., 2005) e são descritos como detentores de atividades anti-HIV e citotóxica em algumas células tumorais, além de inibidores de HIV protease, agente tripanossomicida e inibidor da enzima acetilcolinesterase (Leite et al., 2001; Ma et al., 2005; Barbosa-Filho et al., 2006a).

No gênero Eugenia, foram encontrados relatos de avaliação de atividade moluscicida com os extratos etanólicos das folhas das espécies $E$. dysenterica DC., e E. uniflora L., sendo a primeira ativa a 100 ppm (Bezerra et al., 2002), enquanto a segunda mostrou-se inativa (Luna et al., 2005). Quanto à avaliação da atividade frente às larvas do Aedes aegypti, as espécies E. uniflora (Luna et al., 2005) e E. caryophyllata Thunb. (Syzigium aromaticum) (Costa et al., 2005) mostraram-se ativas.

Nos testes com o espécime de E. malaccensis nativo de Alagoas, os ensaios com as larvas de $A$. aegypti demonstraram que os extratos etanólicos das cascas do caule e das folhas são inativos. Porém, contra os caramujos adultos, esses mesmos extratos demonstraram

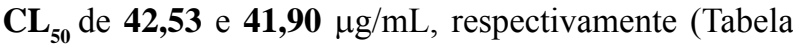
1).

\section{AGRADECIMENTOS:}

Os autores agradecem ao Conselho Nacional de Desenvolvimento Científico e Tecnológico - CNPq, 
Comissão de Aperfeiçoamento de Pessoal de Nível Superior - CAPES e Fundação de Amparo a Pesquisa do Estado de Alagoas - FAPEAL pelo suporte financeiro e pela bolsa de estudo concedida a Anderson Marques de Oliveira. À Prof ${ }^{a}$ Marília Oliveira Fonseca Goulart, ao Programa CAPES/COFECUB e à École Normale Superieure, Paris, pela obtenção dos espectros de RMN. O presente trabalho é parte da dissertação de mestrado de Anderson Marques de Oliveira.

\section{REFERÊNCIAS}

Alberton JR, Ribeiro A, Sacramento LVS, Franco SL, Lima MAP 2001. Caracterização farmacognóstica do jambolão (Syzygium cumini (L.) Skeels). Rev Bras Farmacogn 11: 37-50.

Auricchio MT, Bacchi EM 2003. Folhas de Eugenia uniflora L. (pitanga): propriedades farmacobotânicas, químicas e farmacológicas. Rev Inst Adolfo Lutz 62: 55-61.

Barbosa WLR, Peres A, Gallori S, Vincieri FF 2006. Determination of myricetin derivatives in Chrysobalanus icaco L. (Chrysobalanaceae). Rev Bras Farmacogn 16: 333-337.

Barbosa-Filho JM, Vasconcelos THC, Alencar AA, Batista LM, Oliveira RAG, Guedes DN, Falcão HS, Moura MD, Diniz MFFM, Modesto-Filho J 2005. Plants and their active constituents from South, Central, and North America with hypoglycemic activity. Rev Bras Farmacogn 15: 392-413.

Barbosa-Filho JM, Medeiros KCP, Diniz MFFM, Batista LM, Athayde-Filho PF, Silva MS, Cunha EVL, Almeida JRGS, Quintans-Júnior LJ 2006a. Natural products inhibitors of the enzyme acetylcholinesterase. Rev Bras Farmacogn 16: 258-285.

Barbosa-Filho JM, Martins VKM, Rabelo LA, Moura MD, Silva MS, Cunha EVL, Souza MFV, Almeida RN, Medeiros IA 2006b. Natural products inhibitors of the angiotensin converting enzyme (ACE). A review between 1980-2000. Rev Bras Farmacogn 16: 421446.

Bezerra JCB, Silva IA, Ferreira HD, Ferri PH, Santos SC 2002. Molluscicical activity against Biomphalaria glabrata of Brazilian cerrado medicinal plants. Fitoterapia 73: 428-430.

Campelo CR 1988. Contribuição ao estudo das plantas medicinais no Estado de Alagoas V. Acta-Amazonica 18(Supl): 305-312.

Carvalho JM, Mendonça RPC, Lima MRF, Santos AF, Sant'Ana, AEG 2000. Avaliação da atividade moluscicida de coumarinas, quinonas, fenóis, terpenóides, cetonas, ácidos e aldeídos benzóicos. Resumos da $23^{a}$ Reunião Anual da $S B Q, \mathrm{QB}$ - 028, Poços de Caldas (MG).

Consolini AE, Baldini OAN, Amat AG 1999. Pharmacological basis for the empirical use of Eugenia uniflora L. (Myrtaceae) as antihypertensive. J Ethnopharmacol 66: 33-39.

Corrêa MP 1984. Dicionário das plantas úteis do Brasil e das exóticas cultivadas. Brasília: Ministério da Agricultura-Instituto Brasileiro de Desenvolvimento Florestal, vol. II, p 427.

Costa JGM, Rodrigues FFG, Angélico EC, Silva MR, Mota ML, Santos NKA, Cardoso ALH, Lemos TLG 2005.
Estudo químico-biológico dos óleos essenciais de Hyptis martiusii, Lippia siloides e Syzigium aromaticum frente às larvas do Aedes aegypti. Rev Bras Farmacogn 15: 304-309.

David JP, Meira M, David JM, Guedes MLS 2004. Triterpenos e ferulatos de alquila de Maprounea guianensis. Quim Nova 27: 62-65.

De Almeida SCX, De Lemos TLG, Silveira ER, Pessoa ODL 2005. Constituintes voláteis e não-volatéis de Cachlospermum vitifolium (Willdenow) Sprengel. Quim Nova 28: 57-60.

De Oliveira RN, Dias IJM, Câmara CAG 2005. Estudo comparativo do óleo essencial de Eugenia punicifolia (HBK) DC. de diferentes localidades de Pernambuco. Rev Bras Farmacogn 15: 39-43.

Dos Santos AF, Sant'Ana AEG 1999. Molluscicidal activity of the diterpenoids jatrophone and jatropholones $\mathrm{A}$ and $\mathrm{B}$ isolated from J. elliptica (Pohl) Muell. Arg. Phytother Res 13: 660-664.

Dunstan CA, Noreen Y, Serrano G, Cox PA, Perera P, Bohlin L 1997. Evaluation of some Samoan and Peruvian medicinal plants by prostaglandin biosynthesis and rat ear oedema assays. J Ethnopharmacol 57: 35-56.

EPA 2004. http: //www.epa.gov/nerleerd/stat2.htm\#probit, acessado em Setembro 2004.

Falcão HS, Lima IO, Santos VL, Dantas HF, Diniz MFFM, Barbosa-Filho JM, Batista LM 2005. Review of the plants with anti-inflammatory activity studied in Brazil. Rev Bras Farmacogn 15: 381-391.

Frighetto N, Welendorf RM, Silva AMP, Nakamura MJ, Siani AC 2005. Aplicação de cromatografia centrífuga de contra-corrente na purificação de ácido ursólico das folhas de Eugenia brasilensis Lam. Rev Bras Farmacogn 15: 338-343.

Hussein SAM, Hashem ANM, Seliem MA, Lindequist U, Nawwar MAM 2003. Polyoxygenated flavonoids from Eugenia edulis. Phytochemistry 64: 883-889.

Jain N, Yadava R 1994. Perigrinol, a lupane type triterpene from the fruits of Diospyros peregrina. Phytochemistry 35: 1070-1072.

Joly AB 1993. Botânica: introdução à taxonomia vegetal. São Paulo: Companhia Editora Nacional p. 777.

Kelecom A, Rocha MA, Majdalani EC, Gonzalez MS, Mello CB 2002. Novas atividades biológicas em antigos metabólitos: ácido oleanólico e eugenol de Eugenia caryophyllata. Rev Bras Farmacogn 12(Supl.): 7071.

Kuo P-L 2005. Myricetin inhibits the induction of anti-Fas IgM-, tumor necrosis factor- $\alpha$ - and interleukin$1 \beta$-mediated apoptosis by Fas pathway inhibition in human osteoblastic cell line MG-63. Life Sci 77: 2964-2976.

Kuskoski EM, Vega JM, Rios JJ, Fett R, Troncoso AM, Asuero AG 2003. Characterization of anthocyanins from the fruits of baguaçu (Eugenia umbelliflora Berg). $J$ Agric Food Chem 51: 5450-5454.

Leite JPV, Lombardi JA, Chiari E, Oliveira AB 2001. Isolamento biomonitorado de uma substância tripanossomicida de Arrabidaea triplinervia (Bignoniaceae), o ácido ursólico. Rev Bras Farmacogn 11: 77-87.

Lima, IO, Oliveira RAG, Lima EO, Farias NMP, Souza EL 2006. Atividade antifúngica de óleos essenciais sobre espécies de Candida. Rev Bras Farmacogn 16: 197- 
201.

Limberger RP, Sobral M, Henriques AT, Menut C, Bessière JM 2004. Óleos voláteis de espécies de Myrcia nativas do Rio Grande do Sul. Quim Nova 27: 916-919.

Locher CP, Burch MT, Mower HF, Berestecky J, Davis H, Van Poel B, Lasure A, Vanden Berghe DA, Vlietinck AJ 1995. Anti-microbial activity and anti-complement activity of extracts obtained from selected Hawaiian medicinal plants. J Ethnopharmacol 49: 23-32.

Locher CP, Witvrouw M, Bethune MP, Burch MT, Mower HF, Davis H, Lasure A, Pauwels R, Clercq E, Vlietinck AJ 1996. Antiviral activity of Hawaiian medicinal plants against human immunodeficiency virus type-1 (HIV1). Phytomedicine 2: 259-264.

Lowry JB 1968. The distribution and potential taxonomic value of alkylated ellagic acids. Phytochemistry 7: 18031813.

Lowry JB 1976. Anthocyanins of the Melastomataceae, Myrtaceae and some allied families. Phytochemistry 15: 513-516.

Luna JS, Santos AF, Lima MRF, Omena MC, Mendonça FAC, Bieber LW, Sant'Ana AEG 2005. A study of the larvicidal and molluscicidal activities of some medicinal plants from northeast Brazil. $J$ Ethnopharmacol 97: 199-206.

Lunardi I, Peixoto JLB, Silva CC, Shuquel ITA, Basso EA, Vidotti GJ 2001. Triterpenic acids from Eugenia moraviana. J Braz Chem Soc 12: 180-183.

Ma C-M, Cai S-Q, Cui J-R, Wang R-Q, Tu P-F, Hatorri M, Daneshtalab M 2005. The cytotoxic activity of ursolic acid derivatives. Eur J Med Chem 40: 582-589.

Mahmoud II, Marzouk MSA, Moharram FA, El-Gindi MR, Hassan AMK 2001. Acylated flavonol glycosides from Eugenia jambolana leaves. Phytochemistry 58: 1239-1244.

Negi AS, Darokar MP, Chattopadhyay SK, Garg A, Bhattacharya AK, Srivastava V, Khanuja SPS 2005. Synthesis of a novel plant growth promoter from gallic acid. Bioorg Med Chem Lett 15: 1243-1247.

Noreen Y, Serrano G, Perera P, Bohlin L 1998. Flavan-3-ols isolated from some medicinal plants inhibiting COX1 and COX-2 catalysed prostaglandin biosynthesis. Planta Med 64: 520-524.

Oliveira RN, Dias IJM, Câmara CAG 2005. Estudo comparativo do óleo essencial de Eugenia punicifolia (HBK) DC. de diferentes localidades de Pernambuco. Rev Bras Farmacogn 15: 39-43.

Ong KC, Khoo HE 1997. Review: biological effects of myricetin. Gen Pharmacol 29: 121-126.

Pellegrina CD, Padovani G, Mainente F, Zoccatelli G, Bissoli G, Mosconi S, Veneri G, Peruffo A, Andrighetto G, Rizzi C, Chignola R 2005. Anti-tumour potential of a gallic acid-containing phenolic fraction from Oenothera biennis. Cancer Lett 226: 17-25.

Pepato MT, Mori DM, Baviera AM, Harami JB, Vendramini RC, Brunetti IL 2005. Fruit of the jambolan tree (Eugenia jambolana Lam.) and experimental diabetes. J Ethnopharmacol 96: 43-48.

Pino AP, Marbot R, Rosado A, Vázquez C 2004. Volatile constituents of Malay rose apple [Syzygium malaccense (L.) Merr. \& Perry]. Flavour Frag J 19: 32-35.

Ravi K, Rajasekaran S, Subramanian S 2005. Antihyperlipidemic effect of Eugenia jambolana seed kernel on streptozotocin-induced diabetes in rats. Food Chem Toxicol 43: 1433-1439.

Ruiz ALTG, Magalhães EG, Magalhães AF, Faria AD, Amaral MCE, Serrano DR, Zanotti-Magalhães EM, Magalhães LA 2005. Avaliação da atividade tóxica em Artemia salina e Biomphalaria glabrata de extratos de quatro espécies do gênero Eleocharis (Cyperaceae). Rev Bras Farmacogn 15: 98-102.

Santos GG, Alves JCN, Rodilla JML, Duarte AP, Lithgow AM, Urones JG 1997. Terpenoids and other constituents of Eucalyptus globules. Phytochemistry 44: 1309-1312.

Sharaf M, El-Ansari MA, Saleh NAM 1999. Flavone glycosides from Mentha longifolia. Fitoterapia 70: 478-483.

Silva CV, Bilia DAC, Barbedo CJ 2005a. Fracionamento e germinação de sementes de Eugenia. Rev Bras Semen 27: 86-92.

Silva DA, Costa DA, Silva DF, Souza MFV, Agra MF, Medeiros IA, Barbosa-Filho JM, Braz-Filho R 2005b. Flavonóides glicosilados de Herissantia tiubae (K. Schum) Brizicky (Malvaceae) e testes farmacológicos preliminares do canferol 3,7-di-O- $\alpha$ -L-ramnopiranosídeo. Rev Bras Farmacogn 15: 2329.

Silverstein RM, Brasser GC 1994. Identificação espectrométrica de compostos orgânicos. 5a ed. São Paulo: Editora Guanabara Koogan S.A.

Simas NK, Lima EC, Conceição SR, Kuster RM, OliveiraFilho AM 2004. Produtos naturais para controle da transmissão da dengue - atividade larvicida de Myroxylon balsamum (óleo vermelho) e de terpenóides e fenilpropanóides. Quim Nova 27: 46-49.

Takeda ATC, Breitmaier E, Schenkel, EP 2004. Triterpenes and triterpenoidal glycosides from the fruits of Ilex paraguariensis (mate). J Braz Chem Soc 15: 205211

Timbola AK, Szpoganicz B, Branco A, Monache FD, Pizzolatti MG 2002. A new flavonol from leaves of Eugenia jambolana. Fitoterapia 73: 174-176.

Velázquez E, Tournier HA, Buschiazzo PM, Saavedra G, Schinella GR 2003. Antioxidant activity of Paraguayan plant extracts. Fitoterapia 74: 91-97.

Vendruscolo GS, Rates SMK, Mentz LA 2005. Dados químicos e farmacológicos sobre as plantas utilizadas como medicinais pela comunidade do bairro Ponta Grossa, Porto Alegre, Rio Grande do Sul. Rev Bras Farmacogn 15: 361-372.

Vieira TR, Barbosa LCA, Maltha CRA, Paula VF, Nascimento EA 2004. Constituintes químicos de Malaleuca alternifolia (Myrtaceae). Quim Nova 27: 536-539.

WHO, 1975. World Health Organization. Instruction for determining the susceptibility or resistence of mosquito larvae to insecticides. WHO/VBC/75.583.

Zhang P, Feng Z, Wang Y 2005. Flavonoids, including an unusual flavonoid- $\mathrm{Mg}^{2+}$ salt, from roots of Cudrania cochinchinensis. Phytochemistry 66: 2759-2765. 